\title{
Optimal Pricing Strategies for Bundled Channel in a Smart Phone Supply Chain
}

\author{
Pradipta Patra \\ Woxsen School of Business \\ Hyderabad, Telangana, India. \\ E-mail: pradipdip1@gmail.com / Pradipta.patra@woxsen.edu.in
}

\begin{abstract}
We consider a smart phone supply chain in which customers purchase handsets and service as a bundled package. In this bundled channel, we consider three power structures- manufacturer Stackelberg leader, service operator Stackelberg leader, both manufacturer and service operator decide simultaneously. For each power structure, we study optimal decision of the manufacturer - retail price of smart phone and optimal decision of telecom service operator service price of smart phone. Previous studies have assumed that "service price" is fixed, and service operator decides "subsidy amount" to be paid to the manufacturer. In our work, we relax this assumption and present some new insights. If the subsidy amount (paid by service operator to manufacturer) and service cost (incurred by service operator) are high, the manufacturer sets low retail price for smart phone hand set. If primary demand (for smart phones), subsidy amount and service cost are high, the service operator sets high service price.
\end{abstract}

Keywords: Supply Chain Management; Pricing; Service Operations; Power Structure; Game Theory.

\section{INTRODUCTION AND}

\section{LITERATURE REVIEW}

In a smart phone supply chain the manufacturer and service provider need to complement each other. A telecom service operator cannot provide quality service unless the manufacturer provides handsets of supreme quality. Similarly innovative features developed by smart phone manufacturers cannot function without good quality service provided by operator. Unlike traditional service or product supply chains, the smart phone supply chain delivers both service and product to end customer. So the smart phone supply chain is worth studying and has received considerable attention in supply chain literature.

Customers looking for smart phones and services may purchase handsets from manufacturers and services from telecom service operators separately. In such a scenario the manufacturer works with several service providers and this has been named as "free channel" by Chen and Wang (2015). Alternatively, they can buy handsets and services together as a bundled package from manufacturers or telecom service operators or third party smart phone retailers. This is a "bundled channel" where smart phones are designed to function exclusively for a particular service operator. For example: in United States, a consumer can buy iPhones from Apple stores either as a standalone piece or bundled with a service (Chen and Wang, 2015). In a free channel the manufacturer earns revenue only from the sale of handsets. On the other hand, in a bundled channel the manufacturer earns revenue from sale of handsets and from subsidy amount paid by telecom service operator. The telecom service operator in both channels earn revenue from service price charged to customer for each handset sold. Figure 1 shows the transactions that take place in free channel. Figure 2 does the same for bundled channel. The bundled channel structure is more complex than the free channel structure due to interactions between "end customer and manufacturer plus service operator" as well as between "manufacturer and service operator". In a bundled channel decisions like price of handset, price of service, subsidy amount depend on supply chain power structures (Choi, 1991; Huang and Li, 2001; Cai et al., 2009): Telecom Service Operator- Stackelberg (TS), Vertical Nash (VN) and Manufacturer- Stackelberg (MS).

Our work is related to Chen and Wang (2015). They studied the smart phone supply chain problem by considering that in a bundled channel, manufacturer decides "smart phone retail price" and service operator decides "subsidy amount" to be paid to manufacturer. Chen and Wang (2015) characterized the optimal decisions of both parties under the three supply chain power structures mentioned in the previous paragraph (TS, VN and MS). Further, they compared free channel profits with bundled channel profits to come up with conditions for choosing a particular channel. Chen and Wang (2015) argued that the service price is relatively more stable than price of smart phone handsets. Consequently it is assumed that service price earned by service operator is fixed. Later in this work, numerical study has been used to analyse the effect of service price on smart phone supply chain profits.

Before going on to describing our contribution, we provide a brief review of relevant literature. Dedrick et al. (2011) study the distribution of value in a mobile phone supply chain. They used product-level data to show that carriers get the greatest value (gross profit) from a handset, 
followed closely by manufacturers with suppliers occupying distant third position. Since our work is related to impacts of power structure on supply chain channel management, we mention the notable works in this area. Choi (1991), Ertek and Griffin (2002), Raju and Zhang (2005), Kolay and Shaffer (2013), Cai et al. (2009), Zhang et al. (2012), Shi et al. (2013) all contributed to this branch of supply chain literature.

Since smartphone handset and telecom service are complementary products it would be beneficial to discuss the latest literature on channel strategy, bundling strategy and pricing strategy for complementary products. Yan and Bandyopadhyay (2011) studied the optimum bundling of product categories and the corresponding pricing strategies that maximize profits. This study was able to demonstrate that highly complementary products need to be bundled together and a relatively lower price should be charged. The model, related insights and examples presented in this study can be used by managers to finalize their bundling strategies. Yang and Ng (2010) studied a mixed bundling problem in wireless telecommunication where the customer has the option to purchase a cellular phone at a discounted price provided they subscribe to a particular service plan. They provide conditions under which mixed bundling outperforms individual sale and pure bundling. There are many instances in which customers subscribe to a service and an associated product under a two part tariff scheme. Ferrer et al. (2010) used dynamic programming methodology to analyse the previous mentioned scenario. Yan et al. (2014) studied the impact of advertising and product complementarity on the successful bundling of products. They show that when the degree of complementarity of two products increases the degree of advertisement needed to promote the bundled products decreases. Khamseh et al. (2014) investigated the pricing of complementary goods in a fuzzy supply chain that consists of two manufacturers and one common retailer. Taleizadeh and Charmchi (2015) used Stackelberg game theory model to analyse pricing decisions in a two-echelon supply chain (one manufacturer, one retailer) with two complementary goods under cooperative advertising (agreement between manufacturer and retailer to share advertising cost at the local level). Esmaeilzadeh and Taleizadeh (2016) is another notable work in this area. Xiao et al. (2013) decided distribution channel strategies for a manufacturer with two complementary products. Table 1 in Yan and Bandyopadhyay (2011) presents further literature on complementary product bundling.

Now we discuss the relevance of our work. Chen and Wang (2015) suggested that analysing the telecom service operator's optimal service price may be of interest. We extend their work by considering that the subsidy amount paid by service operator to manufacturer is fixed, and service price is a decision variable of telecom service operator. We consider the same three power structures for bundled channel and in each case find the optimal smart phone retail price set by the manufacturer and optimal service price set by service operator. We also study the effect of different model parameters on optimal solutions. The notations and other assumptions (like linear demand function; both parties aim to maximize their profits and so on) used here are same as in Chen and Wang (2015). Section 2 below presents the mathematical notations needed and the assumptions made. Section 3 presents the propositions and insights on bundled channel models. We perform numerical study in Section 4. Section 5 concludes the paper. Proof of all theorems are presented in the appendix section at the end.

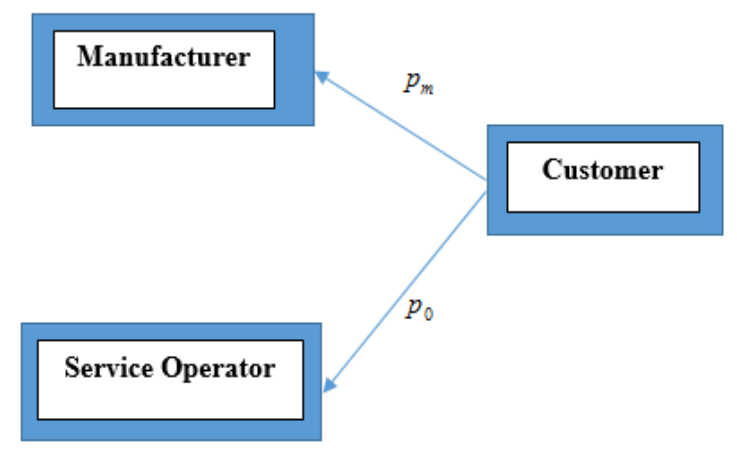

Figure 1 Free Channel Transactions

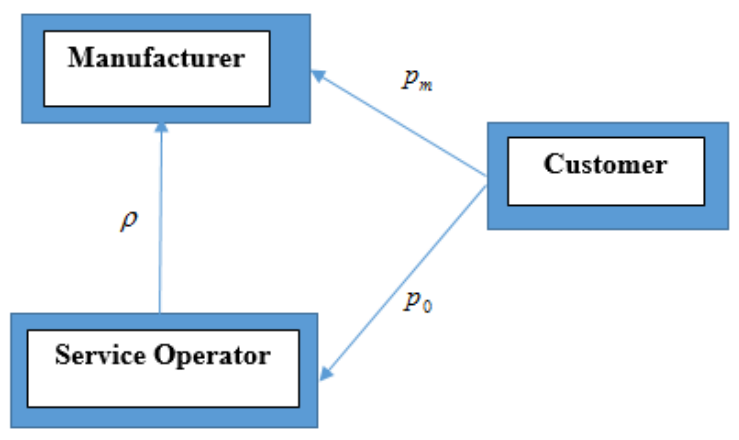

Figure 2 Bundled Channel Transactions

\section{NOTATIONS AND ASSUMPTIONS}

\subsection{Notations}

$p_{m}:$ Unit retail price of smart phone

$c_{m}$ : Unit cost of manufacturing of smart phone

$\rho$ : Subsidy amount paid by telecom service operator to smart phone manufacturer

$p_{0}$ : Unit service price earned by telecom service operator

$c_{0}$ : Unit service cost incurred by telecom service operator

$\alpha$ : Parameter of demand function indicating primary demand of smart phone

$\beta$ : Parameter of demand function indicating customer's sensitivity to retail and service price

$\pi_{m}^{b}($.$) : Manufacturer's profit function in bundled channel$

$\pi_{0}^{b}($.$) : Service operator's profit function in bundled$ channel 


\subsection{Assumptions}

a) Similar to Chen and Wang (2015) we assume that the smart phone supply chain consists of a single upstream manufacturer and single downstream service operator. Chen and Wang (2015) pointed out that this assumption is widely used in supply chain management literature for high-tech products like smart phones. In a bundled channel customers can buy phone and service together from manufacturer or telecom service provider or third party smart phone retailers. Chen and Wang (2015) do not consider the possibility of customers buying from third party smart phone retailers in their model. Instead they focus on the options where both upstream manufacturer and downstream service provider face customers directly. Since our work extends Chen and Wang (2015) we maintain the same assumption. The case where smart phones are sold through third party retailers (for a bundled channel) is interesting and is a potential topic for future research.

b) Similar to Chen and Wang (2015) we assume linear and deterministic demand given by $D\left(p_{m}\right)=\alpha-\beta\left(p_{m}+p_{0}\right)$ where denotes primary demand for smart phones and denotes customers' sensitivity to smart phone retail price.

c) Smart phone manufacturer and telecom service operator aim to maximize their own profits.

d) Smart phone unit retail price $\left(p_{m}\right)$ is more than unit manufacturing cost $\left(c_{m}\right)$. Telecom service operator's unit $\operatorname{price}\left(p_{0}\right)$ is more than unit service $\operatorname{cost}\left(c_{0}\right)$.

e) In a bundled channel the manufacturer earns revenue from sale of handsets and from subsidy amount ( $\rho$ where $-p_{m}<\rho<p_{0}$ ) paid by telecom service operator.

f) The subsidy amount service operator pays to manufacturer is fixed. The service operator decides the optimal service price.

\section{BUNDLED CHANNEL MODEL}

For a bundled channel, equation (1) represents the net profit of smart phone manufacturer. Equation (1) considers the fact that the manufacturer has two sources of revenue: revenue from selling smart phones and subsidy from service operator. Equation (2) gives the net profit of the telecom service operator.

$$
\begin{aligned}
& \pi_{m}^{b}\left(p_{m}\right)=\left(p_{m}-c_{m}+\rho\right)\left[\alpha-\beta\left(p_{m}+p_{0}\right)\right] \\
& \pi_{o}^{b}\left(p_{0}\right)=\left(p_{0}-c_{0}-\rho\right)\left[\alpha-\beta\left(p_{m}+p_{0}\right)\right]
\end{aligned}
$$

\subsection{Telecom Service Operator Stackelberg (TS) Model}

In the TS model, smart phone manufacturer and service operators take decisions in sequence. In this Stackelberg game, service operator is the leader and decides "service price" to be charged to the customer. Smart phone manufacturer is the follower and decides "smart phone retail price". First manufacturer decides "smart phone retail price" for a given "service price". Then, service operator considers manufacturer's response function and decides optimal service price. Proposition 1 below demonstrates that in a bundled channel under TS model, manufacturer's optimal retail price and service operator's optimal service price are existent and unique.

Proposition 1. In a bundled channel under TS power structure,

$$
\begin{aligned}
& p_{m}^{t}=\frac{\alpha}{4 \beta}-\rho-\frac{c_{0}}{4}+\frac{3 c_{m}}{4} \text { and } \\
& p_{0}^{t}=\frac{\alpha}{2 \beta}+\rho+\frac{c_{0}}{2}-\frac{c_{m}}{2} .
\end{aligned}
$$

Insight 1. Optimal retail price $p_{m}^{t}$ is increasing function of $c_{m}$ and $\alpha$, and is a decreasing function of $\rho, c_{0}$ and $\beta$. Optimal service price $p_{0}^{t}$ is increasing function of $\alpha, \rho$ and $c_{0}$, and is a decreasing function of $\beta$ and $c_{m}$.

Insight 1 shows that, as the unit cost of manufacturing of smart phone increases, the optimal retail price also increases. This increase in retail price is because the manufacturer wants to make up the increase in cost via higher revenue. Also, as the primary demand goes up, the optimal retail price also goes up. If customer's sensitivity to smart phone retail price is high, then optimal retail price is low. These insights are same as in the paper by Chen and Wang (2015). The new insights from our work are as follows: as subsidy paid by service operator to manufacturer increases, the optimal smart phone retail price decreases; as service operator's service cost increases, the optimal smart phone retail price decreases.

As telecom service operator's service cost increases, the optimal service price increases. Also as subsidy paid to manufacturer increases, the optimal service price increases. Further, increase in primary demand causes optimal service price to increase. If customer's sensitivity to service price is high, then optimal service price is low. Again as unit cost of manufacturing smart phone increases, the optimal service price decreases. The effect of different model parameters on optimal decisions is depicted graphically in the numerical study section.

\subsection{Vertical Nash (VN) Model}

In this VN model, the smart phone manufacturer and telecom service operator make simultaneous decisions. Proposition 2 below demonstrates that in a bundled channel under VN model, manufacturer's optimal retail price and service operator's optimal service price are existent and unique.

Proposition 2. In a bundled channel under VN power structure,

$$
\begin{aligned}
& p_{m}^{v}=\frac{\alpha}{3 \beta}-\rho-\frac{c_{0}}{3}+\frac{2 c_{m}}{3} \text { and } \\
& p_{0}^{v}=\frac{\alpha}{3 \beta}+\rho+\frac{2 c_{0}}{3}-\frac{c_{m}}{3} .
\end{aligned}
$$


Insight 2. Optimal retail price $p_{m}^{v}$ is increasing function of $c_{m}$ and $\alpha$, and is a decreasing function of $\rho, c_{0}$ and $\beta$. Optimal service price $p_{0}^{v}$ is increasing function of $\alpha, \rho$ and $c_{0}$, and is a decreasing function of $\beta$ and $c_{m}$.

If unit cost of manufacturing of smart phone and primary demand are high, the optimal retail price of smart phone is also high. If customer's sensitivity to retail price of smart phone, service cost of operator and subsidy from operator are high, then smart phone manufacturer sets low retail price.

If customer's sensitivity to service price of smart phone and unit cost of manufacturing smart phone are high, then telecom service operator sets low service price. If primary demand, subsidy paid to manufacturer, service cost of the operator are high, the service operator sets high service price. Please refer to the numerical study section to view (graphically) the effect of model parameters on optimal decisions.

\subsection{Manufacturer Stackelberg (MS) Model}

In the MS model, smart phone manufacturer and service operators take decisions in sequence. In this Stackelberg game, service operator is the follower and decides "service price" to be charged to the customer. Smart phone manufacturer is the leader and decides "smart phone retail price". Proposition 3 below demonstrates that in a bundled channel under MS model, manufacturer's optimal retail price and service operator's optimal service price are existent and unique.

Proposition 3. In a bundled channel under MS power structure,

$$
\begin{aligned}
& p_{m}^{m}=\frac{\alpha}{2 \beta}-\rho+\frac{c_{m}}{2}-\frac{c_{0}}{2} \text { and } \\
& p_{0}^{m}=\frac{\alpha}{4 \beta}+\rho+\frac{3 c_{0}}{4}-\frac{c_{m}}{4} .
\end{aligned}
$$

Insight 3. Optimal retail price $p_{m}^{m}$ is increasing function of $c_{m}$ and $\alpha$, and is a decreasing function of $\rho, c_{0}$ and $\beta$. Optimal service price $p_{0}^{m}$ is increasing function of $\alpha, \rho$ and $c_{0}$, and is a decreasing function of $\beta$ and $c_{m}$.

If unit cost of manufacturing of smart phone and primary demand are high, the optimal retail price of smart phone is also high. If customer's sensitivity to retail price of smart phone, service cost of operator and subsidy from operator are high, then smart phone manufacturer sets low retail price.

If customer's sensitivity to service price of smart phone and unit cost of manufacturing smart phone are high, then telecom service operator sets low service price. If primary demand, subsidy paid to manufacturer, service cost of the operator are high, the service operator sets high service price. The effect of different model parameters on optimal decisions can be seen graphically in the following section.

\section{NUMERICAL STUDY}

In this section we use numerical study to present the effect of different model parameters on the optimal service price and optimal retail price of smart phones. For the purpose of our study we use $\alpha=100 ; \beta=0.1 ; c_{m}=100 ; c_{0}=50$ as the fixed parameter values. Figure 3 below displays the effect of subsidy amount ( $\rho$ paid by telecom service operator to manufacturer) on "optimal retail price of smart phone" and "optimal service price" as given in Proposition1. Figure 3 shows that as the "subsidy amount" increases the telecom service operator charges a higher service price but the manufacturer charges a lower retail price for the smart phone. Since the effect of subsidy amount on optimal decisions remain same in Proposition 2 and Proposition 3 we do not display them graphically.

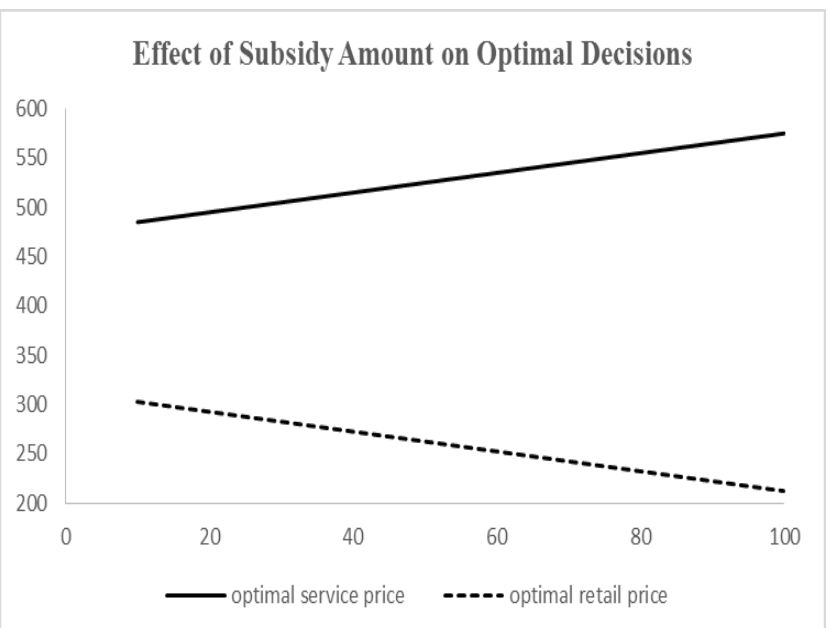

Figure 3 Subsidy amount vs optimal retail price and optimal service price

Next we study the effect of smart phone manufacturing $\operatorname{cost}\left(c_{m}\right)$ on optimal decisions - service price and retail price. For this study we fix parameter values as $\alpha=100 ; \beta=0.1 ; \rho=100 ; c_{0}=50$. Again we make use of Proposition 1 and display the results in Figure 4 below. Figure 4 shows that the optimal retail price of smart phone increases as the manufacturing cost increases but the optimal service price decreases. These insights extend to Proposition 2 and Proposition 3. Hence we do not display them graphically.

Finally we study the effect of service $\operatorname{cost}\left(c_{0}\right)$ on the two optimal decisions. We use $\alpha=100 ; \beta=0.1 ; \rho=50 ; c_{m}=100$ as the fixed value of the parameters. Figure 5 below presents the insights from Proposition 1. As the cost of service increases the optimal service price charged by the provider increases but the retail price charged by the manufacturer decreases. Once again we do not display Propositions 2 and 3 graphically. 


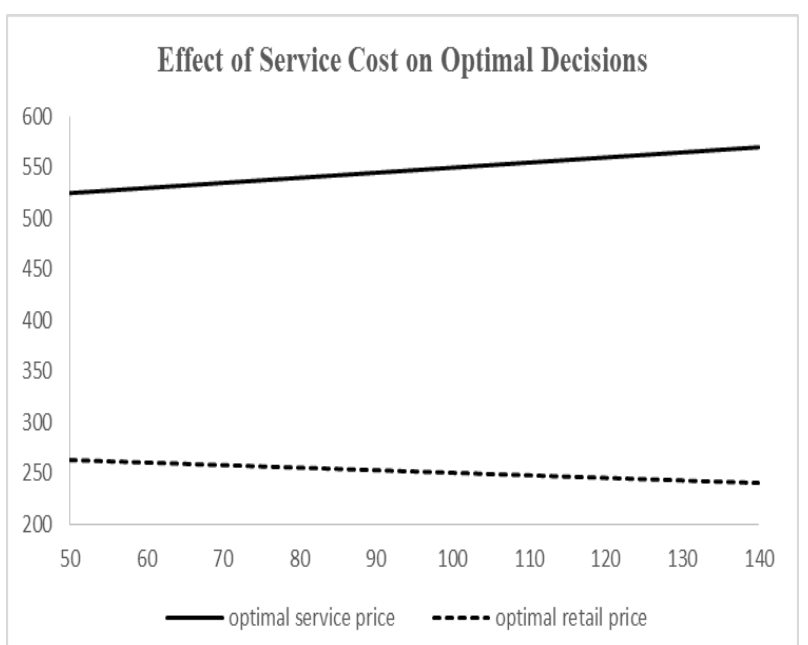

Figure 4 Manufacturing cost vs optimal retail price and optimal service price

\section{CONCLUSION}

In this paper, we have extended the work done by Chen and Wang (2015). They considered free channel models and bundled channel models for a smart phone supply chain. For bundled channel models, they considered "smart phone retail price" as manufacturer's decision variable and "subsidy amount" as service operator's decision variable. We have considered "service price" as the decision variable of service operator and reworked the propositions of the original paper. We have been able to show that the optimal service price under different supply chain power structures exists. We have been able to study
Operations and Supply Chain Management 9(3) pp. 199 - 205 (02016

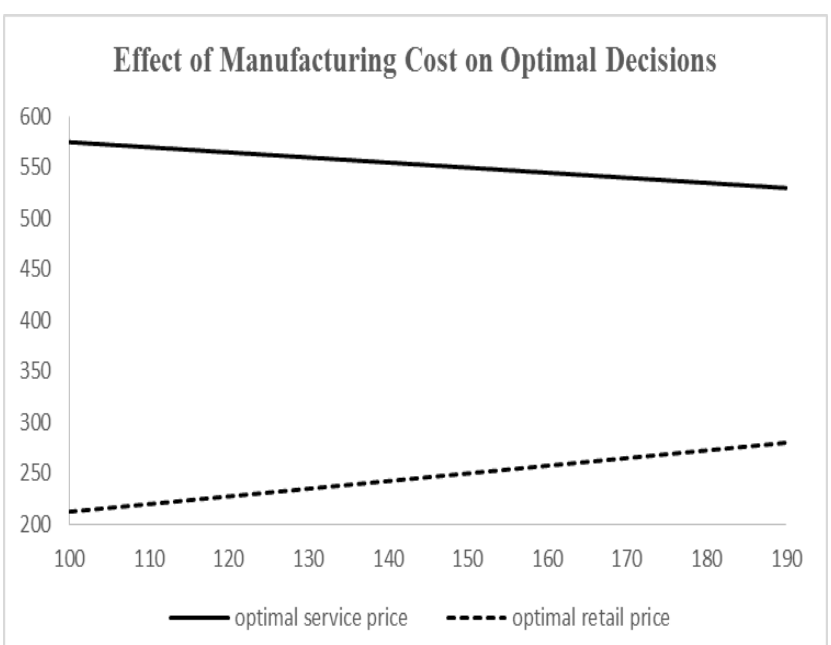

Figure 5 Service cost vs optimal retail price and optimal service price

the effect of different model parameters on optimal service price. Factors like primary demand for smart phone, the subsidy amount paid to manufacturer, service cost incurred by the service operator positively affects optimal service price. Cost of manufacturing smart phones and sensitivity of customer (to service price of smart phones) negatively affects optimal service price. Table 1 below summarizes the results of our work.

We have assumed in our work that end customers purchase the bundled products either from manufacturer or service provider. The case where bundled products is purchased from third party retailers is interesting and provides scope for future research.

Table 1 Summary of results

\begin{tabular}{|c|c|c|}
\hline Power Structure & Chen and Wang (2015) & Our Results \\
\hline TS & $p_{m}^{t}=\frac{3 \alpha}{4 \beta}-p_{0}+\frac{c_{0}}{4}+\frac{c_{m}}{4}$ & $p_{m}^{t}=\frac{\alpha}{4 \beta}-\rho-\frac{c_{0}}{4}+\frac{3 c_{m}}{4}$ \\
& $\rho^{t}=p_{0}-\frac{c_{0}}{2}+\frac{c_{m}}{2}-\frac{\alpha}{2 \beta}$ & $p_{0}^{t}=\frac{\alpha}{2 \beta}+\rho+\frac{c_{0}}{2}-\frac{c_{m}}{2}$ \\
\hline VN & $p_{m}^{v}=\frac{2 \alpha}{3 \beta}-p_{0}+\frac{c_{0}}{3}+\frac{c_{m}}{3}$ & $p_{m}^{v}=\frac{\alpha}{3 \beta}-\rho-\frac{c_{0}}{3}+\frac{2 c_{m}}{3}$ \\
\hline MS & $\rho^{v}=p_{0}-\frac{2 c_{0}}{3}+\frac{c_{m}}{3}-\frac{\alpha}{3 \beta}$ & $p_{0}^{v}=\frac{\alpha}{3 \beta}+\rho+\frac{2 c_{0}}{3}-\frac{c_{m}}{3}$ \\
\hline$p_{m}^{m}=\frac{3 \alpha}{4 \beta}-p_{0}+\frac{c_{m}}{4}+\frac{c_{0}}{4}$ & $p_{m}^{m}=\frac{\alpha}{2 \beta}-\rho+\frac{c_{m}}{2}-\frac{c_{0}}{2}$ \\
& $\rho^{m}=p_{0}-\frac{3 c_{0}}{4}+\frac{c_{m}}{4}-\frac{\alpha}{4 \beta}$ & $p_{0}^{m}=\frac{\alpha}{4 \beta}+\rho+\frac{3 c_{0}}{4}-\frac{c_{m}}{4}$ \\
\hline
\end{tabular}




\section{REFERENCES}

Cai, G.G., Zhang Z.G., and Zhang, M. (2009). Game theoretical perspectives on dual-channel supply chain competition with price discounts and pricing schemes. International Journal of Production Economics 117(1), pp. 80-96.

Chen, X., and Wang, X. (2015). Free or bundled: Channel selection decisions under different power structures. OMEGA- the International Journal of Management Science 53, pp. 11-20

Choi SC. (1991). Price competition in a channel structure with a common retailer. Marketing Science 10(4), pp. 271-296.

Dedrick J., Kraemer K.L., and Linden G. (2011). The distribution of value in the mobile phone supply chain. Telecommunications Policy 35(6), pp. 505-521.

Ertek, G., and Griffin, P.M. (2002). Supplier and buyer driven channels in a two-stage supply chain. IIE Transactions 34(8), pp. 691-700.

Esmaeilzadeh, A., and Taleizadeh, A.A. (2016). Pricing in a twoechelon supply chain with different market powers: game theory approaches. Journal of Industrial Engineering International 12(1), pp. 119-135.

Ferrer, J.C., Mora, H., and Olivares, F. (2010). On pricing of multiple bundles of products and services. European Journal of Operational Research 206(1), pp. 197-208.

Huang, Z., and Li, SX. (2001). Co-op advertising models in manufacturer-retailer supply chains: a game theory approach. European Journal of Operational Research 135(3), pp. 527-544.

Khamseh, A.A., Soleimani, F., and Naderi, B. (2014). Pricing decisions for complementary products with firm's different market powers in fuzzy environments. Journal of Intelligent \& Fuzzy Systems 27(5), pp. 2327-2340.

Kolay, S., and Shaffer, G. (2013). Contract design with a dominant retailer and a competitive fringe. Management Science 59(9), pp. 2111-2116.

Raju, J., and Zhang, Z.J. (2005). Channel coordination in the presence of a dominant retailer. Marketing Science 24(2), pp. 254-262.

Shi, R., Zhang, J., and Ru, J. (2013). Impacts of power structure on supply chains with uncertain demand. Production and Operations Management 22(5), pp. 1232-1249.

Taleizadeh, A.A., and Charmchi, M. (2015). Optimal advertising and pricing decisions for complementary products. Journal of Industrial Engineering International 11(1), pp. 111-117.

Xia, Y., Xiao, T. and Zhang, G. P. (2013). Distribution Channel Strategies for a Manufacturer with Complementary Products. Decision Sciences 44(1), pp. 39-56.

Yan, R., and Bandyopadhyay, S. (2011). The profit benefits of bundle pricing of complementary products. Journal of Retailing and Consumer Services 18(4), pp. 355-361.

Yan, R., Myers, C., Wang, J., and Ghose, S. (2014). Bundling products to success: The influence of complementarity and advertising. Journal of Retailing and Consumer Services 21(1), pp. 48-53.

Yang, B., and Ng, C.T. (2010). Pricing problem in wireless telecommunication product and service bundling. European Journal of Operational Research 207(1), pp. 473-480.

Zhang, R., Liu, B., and Wang, W. (2012). Pricing decisions in a dual channels system with different power structures. Economic Modelling 29(2), pp. 523-533.

\section{APPENDIX}

\section{Proof of Proposition 1:}

Differentiating Equation (1) twice w.r.t. $p_{m}$ we get,

$$
\frac{\partial \pi_{m}^{b}\left(p_{m}\right)}{\partial p_{m}}=\left\{\alpha-\beta\left(p_{m}+p_{o}\right)\right\}+\left\{p_{m}+\rho-c_{m}\right\} \times(-\beta)
$$

$\frac{\partial^{2} \pi_{m}^{b}\left(p_{m}\right)}{\partial p_{m}^{2}}=2 \times(-\beta)<0$.

So $\pi_{m}^{b}\left(p_{m}\right)$ is concave function of $p_{m}$. From first order condition (FOC) we get $p_{m}=\frac{\alpha}{2 \beta}-\frac{p_{o}}{2}-\frac{\rho}{2}+\frac{c_{m}}{2}$.

Substituting this value of $p_{m}$ in equation (2) we get the following equation.

$\pi_{o}^{b}\left(p_{o}\right)=\left(p_{o}-c_{o}-\rho\right)\left(\frac{\alpha-\beta p_{o}+\beta \rho-\beta c_{m}}{2}\right)$.

Differentiating this equation twice w.r.t. $p_{o}$, we can show that $\frac{\partial^{2} \pi_{o}^{b}\left(p_{o}\right)}{\partial p_{o}^{2}}=-\beta<0$ i.e. $\quad \pi_{o}^{b}\left(p_{o}\right)$ is $\quad$ concave function of $p_{o}$. From FOC it can be shown that $p_{o}^{t}=\frac{\alpha}{2 \beta}+\rho+\frac{c_{o}}{2}-\frac{c_{m}}{2}$.

Substituting this $p_{o}^{t}$ in $p_{m}=\frac{\alpha}{2 \beta}-\frac{p_{o}}{2}-\frac{\rho}{2}+\frac{c_{m}}{2}$

we get $p_{m}^{t}=\frac{\alpha}{4 \beta}-\rho+\frac{3 c_{m}}{4}-\frac{c_{o}}{4}$.

Proof of Proposition 2: The initial steps of this proof are same as proposition 1 . We differentiate equation (1) twice w.r.t. $\quad p_{m}$ to $\quad$ get $p_{m}=\frac{\alpha}{2 \beta}-\frac{p_{o}}{2}-\frac{\rho}{2}+\frac{c_{m}}{2}$. The following equations are obtained when we differentiate equation (2) twice w.r.t. $p_{o}$.

$\frac{\partial \pi_{o}^{b}\left(p_{o}\right)}{\partial p_{o}}=\left\{\alpha-\beta\left(p_{m}+p_{o}\right)\right\}+\left\{p_{o}-\rho-c_{o}\right\} \times(-\beta)$ $\frac{\partial^{2} \pi_{o}^{b}\left(p_{o}\right)}{\partial p_{o}^{2}}=2 \times(-\beta)<0$.

So $\pi_{o}^{b}\left(p_{o}\right)$ is concave function of $p_{o}$. From first order condition (FOC) we get $p_{o}=\frac{\alpha}{2 \beta}-\frac{p_{m}}{2}+\frac{\rho}{2}+\frac{c_{o}}{2}$. 
Solving $p_{m}=\frac{\alpha}{2 \beta}-\frac{p_{o}}{2}-\frac{\rho}{2}+\frac{c_{m}}{2}$

and

$p_{o}=\frac{\alpha}{2 \beta}-\frac{p_{m}}{2}+\frac{\rho}{2}+\frac{c_{o}}{2}$ simultaneously and then

simplifying we get the expressions given in Proposition 2.

Proof of Proposition 3: We first differentiate equation (2) twice w.r.t. $p_{o}$.

$\frac{\partial \pi_{o}^{b}\left(p_{o}\right)}{\partial p_{o}}=\left\{\alpha-\beta\left(p_{m}+p_{o}\right)\right\}+\left\{p_{o}-\rho-c_{o}\right\} \times(-\beta)$

$\frac{\partial^{2} \pi_{o}^{b}\left(p_{o}\right)}{\partial p_{o}^{2}}=2 \times(-\beta)<0$.

So $\pi_{o}^{b}\left(p_{o}\right)$ is concave function of $p_{o}$. From first order condition (FOC) we get $p_{o}=\frac{\alpha}{2 \beta}-\frac{p_{m}}{2}+\frac{\rho}{2}+\frac{c_{o}}{2}$.

Substituting this expression of $p_{o}$ in equation (1) we get,

$$
\pi_{m}^{b}\left(p_{m}\right)=\left(p_{m}-c_{m}+\rho\right)\left(\frac{\alpha-\beta p_{m}-\beta \rho-\beta c_{o}}{2}\right)
$$

We can show that $\frac{\partial^{2} \pi_{m}^{b}\left(p_{m}\right)}{\partial p_{m}^{2}}=-\beta<0$ i.e. $\pi_{m}^{b}\left(p_{m}\right)$ is concave function of $p_{m}$. From FOC we get $p_{m}^{m}=\frac{\alpha}{2 \beta}-\rho+\frac{c_{m}}{2}-\frac{c_{o}}{2}$.

Substituting this

value

of $p_{m}^{m}$ in $p_{o}=\frac{\alpha}{2 \beta}-\frac{p_{m}}{2}+\frac{\rho}{2}+\frac{c_{o}}{2}$ we get the expression given in proposition 3 .

Pradipta Patra completed FPM (equivalent to PhD) from IIM Bangalore in 2015. His thesis is titled - "Analysis of Performance-Based Contracts for Capital Equipment and Manufacturing Systems". Post PhD he has been teaching at Woxsen School of Business Hyderabad and IIM Sambalpur. He has taught courses for both 2 year regular program as well as 1 year executive program. He teaches courses like Quantitative Techniques (Probability, Statistics and Operations Research), Operations Management, Operations Strategy, Service Operations Management and Business Forecasting. Pradipta Patra is also interested in doing research in the area of operations management, supply chain management, supply chain contracts, service operations, reliability, maintenance, and risk analysis in supply chain. 